\title{
The role of working memory in analogical mapping
}

\author{
JAMES A. WALTZ, ALBERT LAU, SARA K. GREWAL, and KEITH J. HOLYOAK \\ University of California, Los Angeles, Califormia
}

\begin{abstract}
The impact of a working-memory load on analogical mapping was examined in two experiments, using a dual-task paradigm. In Experiment 1, we used a phonological working-memory load; in Experiment 2, we used a phonological working-memory load and an executive working-memory load. The subjects were required to identify correspondences between visual scenes, either for single objects or for three objects simultaneously. The results indicated that the imposition of either a phonological or an executive working-memory load decreased the frequency with which the subjects identified correspondences between scenes based on relations and increased the frequency with which they identified correspondences based on object attributes. The frequency with which subjects made relational mappings was increased by the instruction to find correspondences for multiple objects in a scene simultaneously, rather than for just one. These results indicate that mapping on the basis of relations places greater demands on both modality-specific buffers and executive components of working memory than does mapping on the basis of object attributes.
\end{abstract}

In humans, mental representations appear to involve different levels of complexity and abstraction. An important type of cognitive process in which representations of varying complexity play major roles is that used to compute similarities and analogical correspondences. Although similarity has sometimes been modeled in terms of overlap between undifferentiated sets of features (Tversky, 1977), there is evidence that similarity judgments also depend on relations between elements (Goldstone, Medin, \& Gentner, 1991; Medin, Goldstone, \& Gentner, 1993). Formally, relational representations have a predicateargument structure, in which one or more of the elements are bound to distinct role(s). Gentner (1983) proposed a taxonomy of representational complexity that ranges from attributes (i.e., one-place predicates, such as tall [Abe]) to first-order relations (i.e., multiplace predicates that take objects as role fillers, such as taller than [Abe, Bill]) to higher order relations (multiplace predicates in which at least one role filler is itself a proposition, such as cause (taller-than [Abe, Bill], jealous-of [Bill, Abe]). Premack (1983) and Halford (1993) have proposed similar representational taxonomies. For the purposes of the present paper, it will suffice to distinguish representations based on the attributes of individual objects from representations based on relations (either first order or higher order) among multiple objects.

This research was supported by NSF Grant SBR-9729023. We thank Art Markman for generously providing us with electronic versions of the pictures used by Markman and Gentner (1993). Dedre Gentner, Steve Sloman, and an anonymous reviewer provided helpful comments on an earlier draft. Experiment 1 was reported at the Twenty-first Annual Conference of the Cognitive Science Society (Vancouver, August 1999). J. Waltz is now at the Neurophysiology Department, Max Planck Institute for Brain Research, Deutschordenstrasse 46, 60528 Frankfurt a.M., Germany (e-mail: waltz@mpih-frankfurt.mpg.de). Requests for reprints may be sent to K. Holyoak, Department of Psychology, UCLA Los Angeles, CA 90095-1563 (e-mail: holyoak@lifesci.ucla.edu).
Both attributes and relations can provide a basis for finding analogical correspondences, or mappings, between situations. In some cases, correspondences based on information at different levels of complexity may conflict, creating ambiguous cross mappings between elements of the two analogs (see, e.g., Gentner \& Toupin, 1986; Ross, 1987, 1989). For example, Markman and Gentner (1993) showed college students pairs of pictures, such as that of a man bringing groceries from a truck and giving them to a woman, who is thanking him, and of a different woman taking food from a bowl and giving it to a squirrel (see Figure 1). The subjects were asked to indicate which object in the second picture corresponded to the woman in the first picture. On the basis of attribute mapping, the woman in the first picture would map to the woman in the second picture; but on the basis of relational mapping, the woman in the first picture would map to the squirrel in the second picture because each is a recipient of the food.

Markman and Gentner (1993) found that different subjects gave different responses to such cross-mapped objects, some giving the attribute-based response and some giving the relation-based response. Manipulations that encouraged the subjects to build an integrated representation of the relations among the objects and of the higher order relations between relations increased the proportion of relational responses. In particular, if subjects were asked to match not just one object in the first picture (the woman), but three (the woman, man, and groceries) to an object in the second picture, they were more likely to map the woman to the squirrel on the basis of their similar relational roles than the subjects who mapped the woman alone. Active mapping of multiple objects seems to encourage people to process relations, which in turn changes the apparent correspondences between individual objects.

Although people are clearly capable of mapping on the basis of multiple levels of representational complexity, 


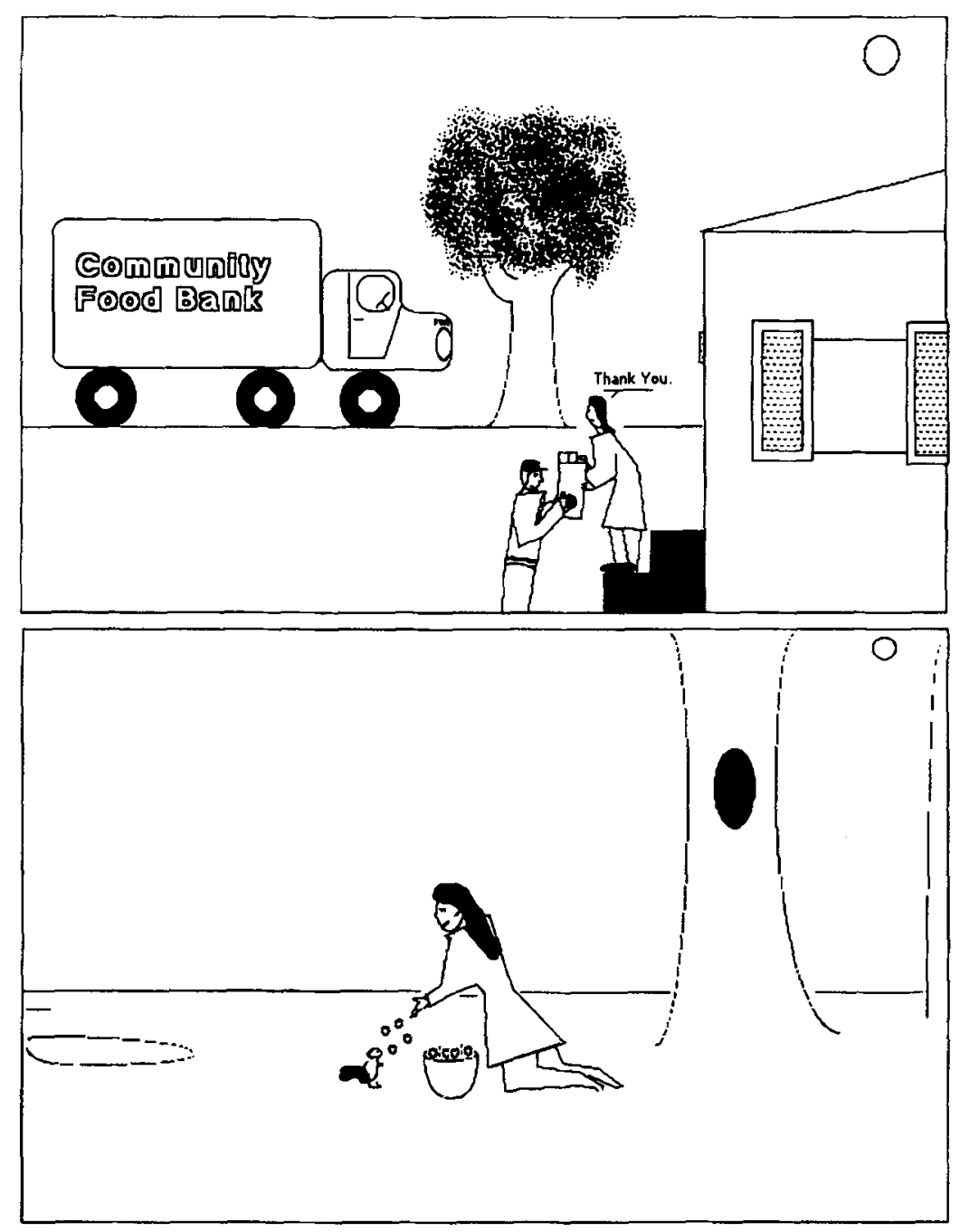

Figure 1. Sample item from mapping task. This item involves a cross-mapping, in that the woman in the illustration at the top can be mapped to the woman in the bottom scene (on the basis of object attributes) or to the squirrel (on the basis of relational similarity, as both fill the role of giver-of-food). Reprinted with permission from Markman and Gentner (1993).

there is reason to expect that relational mapping imposes greater demands on working memory than does attributional mapping (Halford, 1993; see Baddeley, 1986, 1992 , for a discussion of the components of human working memory). For example, the mapping of the woman in the first picture to the woman in the second picture can be done by focusing on only one object in each picture, whereas the mapping of the woman to the squirrel requires the representation of multiple objects and relations in each picture in order to recognize the correspondences between the objects filling matching roles in a system of relations. Some computational models of analogical mapping, such as the STAR model of Halford et al. (1994) and the LISA model of Hummel and Holyoak (1997), postulate that there are inherent limitations on the complexity of possible mappings due to working-memory limits. Such models have led to the general prediction that any manipulation that reduces available working-memory capacity will make it more difficult for reasoners to compute relational mappings, and hence will increase the proportion of less complex attribute mappings in situations in which the mapping is ambiguous.

A number of lines of evidence support the hypothesis that mapping on the basis of relations is more complex than mapping on the basis of attributes. From the standpoint of phylogeny, studies with nonhuman animals indicate that the ability to identify relational correspondences emerges only with great apes (Premack, 1983; Thompson, Oden, \& Boysen, 1997). In human ontogeny, children develop the capacity to map on the basis of attributes at a younger age than that at which they can map 
on the basis of relations, especially higher order relations (Gentner, Rattermann, Markman, \& Kotovsky, 1995; Halford \& Wilson, 1980; Smith, 1989). There is also evidence that the ability to integrate relations on line can be selectively impaired - for example, by damage to the prefrontal cortex in human adults (Waltz et al., 1999).

The results of a number of studies suggest that there is a close relationship between the ability to process multiple relations and the functioning of working memory. Many of these studies have involved the performance of reasoning tasks concurrently with secondary tasks designed to place demands on working-memory systems. In a study in which a dual-task paradigm was used to investigate the information-processing demands of relational reasoning, Maybery, Bain, and Halford (1986) required subjects to respond to a probe at different stages in the solution of three-term series (transitive inference) problems. Maybery et al. found that reaction times to the probe were longest during the phases of the problems in which premise integration took place--that is, during the presentation of the second premise and during the presentation of the proposition to be verified. Maybery et al.'s results were interpreted as indicating that the process of relational premise integration involved greater informationprocessing requirements than did the sequential processing of individual relations, because premise integration requires multiple relations to be simultaneously active in working memory.

Dual-task paradigms have often been used to examine the role of different components of working memory in reasoning. According to Baddeley $(1986,1992)$, working memory is a tripartite system, with an executive control module overseeing access to two "slave" systems for shortterm storage - a phonological loop and a visuospatial scratchpad. Numerous studies have demonstrated the functional independence of these aspects of working memory (Baddeley, 1992) and have led to the development of a set of tasks designed to occupy working memory's specific components. The performance of reasoning tasks concurrently with tasks designed to occupy working memory has made it possible to examine the role of working-memory components in reasoning. Thus Gilhooly, Logie, Wetherick, and Wynn (1993) found that individuals who were required to perform a syllogistic reasoning task while simultaneously performing a task designed to place demands on the executive system of working memory (i.e., attempting to generate random digits) showed significant impairment in the reasoning task. Similar results have been found for tasks involving propositional reasoning (Klauer, Stegmaier, \& Meiser, 1997; Toms, Morris, \& Ward, 1993).

In the present study, college students performed a mapping task in which they were asked to determine correspondences between thematic visual scenes involving cross-mappings while simultaneously performing a secondary task that would tax one or more components of working memory. The central prediction was that imposing a load on working memory would selectively impair the generation of correspondences based on relations, so that relational mappings would be made less frequently and attribute mappings would be made more frequently.

\section{EXPERIMENT 1}

The purpose of Experiment 1 was to examine the effect of a phonological working-memory load on structure mapping. In this experiment, we used materials taken from the study by Markman and Gentner (1993), which provided evidence that structure mapping is involved in similarity comparisons. The working-memory manipulation was modeled after previous studies that used dualtask paradigms to investigate the role of working memory in reasoning. The results of some previous studies of reasoning in which dual-task paradigms were used suggest that the phonological loop of working memory may not figure prominently in propositional reasoning (see, e.g., Gilhooly et al., 1993). However, the reasoning tasks that have been used in such studies might invite the application of heuristics that reduce their working-memory demands (Halford, Bain, \& Maybery, 1984). Theories of relational complexity (Halford, Wilson, \& Phillips, 1998) have posited that when multiple verbal premises must be integrated, the on-line representation of a system of relations places greater demands on phonological working memory than does the representation of individual relations.

Although the stimuli used in the present study were visual, it seemed likely that people would use verbal coding to develop a representation of the abstract relations involved. For example, the "give" relation in the top picture shown in Figure 1 can presumably be inferred in part by reading the phrases included in the picture (Community Food Bank and thank you), after which a verbal description of the scene may be formed. (Seven of the eight picture pairs included words or phrases that helped make the theme understandable.) In fact, the need to actively construct a representation of such abstract relations from pictures may place greater demands on phonological working memory than does processing sentences that state relations directly. Accordingly, we hypothesized that the subjects with reduced working-memory resources due to the imposition of a phonological memory load would be less likely to identify correspondences based on relational similarity.

In Experiment 1, we also examined whether a workingmemory load would have differential effects in conditions in which the subjects were prompted to identify correspondences for single versus multiple objects. Different predictions can be made for this comparison. It might be hypothesized that if working memory primarily influences the process of structure mapping, then the effect of load on the tendency to identify correspondences based on relational similarity would be greater when subjects are prompted to identify correspondences for multiple objects rather than for single objects (since individuals are more likely to engage in a process of structure mapping when prompted to identify correspondences for 
multiple objects). On the other hand, if working-memory capacity is a factor in the initial recognition of the possibility of a relation-based mapping, one might expect a memory load to have a greater effect on the tendency to identify correspondences based on relational similarity when subjects are prompted to identify correspondences between scenarios for single rather than for multiple objects (since the possibility of making a relation-based mapping may be more difficult to recognize when single objects are being mapped). These two alternatives are not mutually exclusive; if both factors are involved, the net result might be a roughly equivalent impact of load on relational mapping for mapping single and multiple objects.

\section{Method}

Subjects. Forty-five undergraduate students from the University of California, Los Angeles served in the experiment as unpaid volunteers.

Materials and Apparatus. Macintosh microcomputers running the SuperLab software package were used to present the stimuli. The stimuli consisted of the eight pairs of pictures that had been used by Markman and Gentner (1993) and eight different 7-digit number strings. Each of the pictures showed a visual scene with three or more objects (see Figure 1 for an example). One of these objects could be cross mapped; that is, it could be judged as corresponding to one object on the basis of physical attributes and to a different object on the basis of the role it played in a system of relations. For instance, the woman in the top picture in Figure 1 could be mapped to the woman in the bottom picture on the basis of physical attributes or to the squirrel on the basis of the fact that the two objects both fill the role of "receiver of food."

Design and Procedure. A $2 \times 2$ between-subjects design was used, with each subject randomly assigned to one of four conditions. The variables manipulated were the number of objects in each picture, for which the subjects were prompted to identify correspondences (one or three), and the presence or absence of a phonological working-memory load. Half of the subjects were assigned to the 1-map condition and half were assigned to the 3-map condition. Orthogonally, half of the subjects in each group were required to hold digit strings in working memory while performing the mapping task, and half were not.

Each subject sat at a computer with an experimenter. Written instructions were displayed on the computer screen, explaining the procedure and the tasks to be performed. The subjects were told that they would be presented with eight pairs of pictures and that each pair would be presented with one picture above the other on a computer screen. For each pair of pictures, the experimenter would point to a set of objects in the first picture. The subject's task was to identify the objects in the second picture that corresponded to each of those indicated by the experimenter. In the 1-map condition, the subjects were prompted to identify the object in the bottom picture corresponding to one object (the cross-mapped one) in the top picture. In the 3-map condition, the subjects were asked to identify the objects in the bottom picture corresponding to three objects in the top picture. The three objects were numbered $(1-3)$. The numbers were shown simultaneously and indicated the required order for mapping the three objects. The first object to be mapped in the 3map condition was the same cross-mapped object that was the single object to be mapped in the 1-map condition.

In the phonological working-memory condition, a string of seven digits was presented visually to the subjects for $4 \mathrm{sec}$, followed by a blank screen that was displayed for $1 \mathrm{sec}$, after which the subjects started the primary task. Although the subjects were not explicitly told to emphasize one task over the other, they were told that they would need to remember the string of digits for testing. After point- ing to their answer(s) in the mapping task, the subjects in this condition were given $5 \mathrm{sec}$ in which to recall the 7-digit number string that was shown prior to the set of visual scenes. The experimenter recorded the subjects' responses to the mapping questions and their responses for the digit-string memory task. All the subjects viewed each of the eight picture pairs once and viewed the pairs in the same order. The subjects controlled the length of time for which visual scenes were displayed.

\section{Results and Discussion}

The dependent variable was the proportion of correspondences identified by the subjects on the basis of relational similarity. The effects of mapping condition (1-map vs. 3-map) and the presence or absence of a working-memory load on the percentage of relational mappings were analyzed with a $2 \times 2$ between-subjects analysis of variance (ANOVA). The means for the four conditions are depicted in Figure 2. The analysis revealed a main effect of mapping instruction such that the mean proportion of relational responses was significantly higher in the 3-map condition than in the 1-map condition $[M=$ .60 vs. $\left.M=.39 ; F(1,41)=6.64, M S_{\mathrm{e}}=.0778, p<.05\right]$. A main effect of working-memory load was also observed: The mean proportion of relational responses was significantly lower when a digit string had to be maintained than when there was no memory load $[M=.35$ vs. $\left.M=.64 ; F(1,41)=12.48, M S_{\mathrm{e}}=.0778, p<.01\right]$. There was no significant interaction between number of mapping questions and memory load $[F(1,41)<1]$.

When the subjects failed to provide a relational mapping, their responses were not simply random; rather, in the vast majority of such cases, the subjects provided mappings on the basis of attributional similarity. Only $7.5 \%$ of mappings made by all the subjects could not be interpreted as either relational or attributional. Overall,

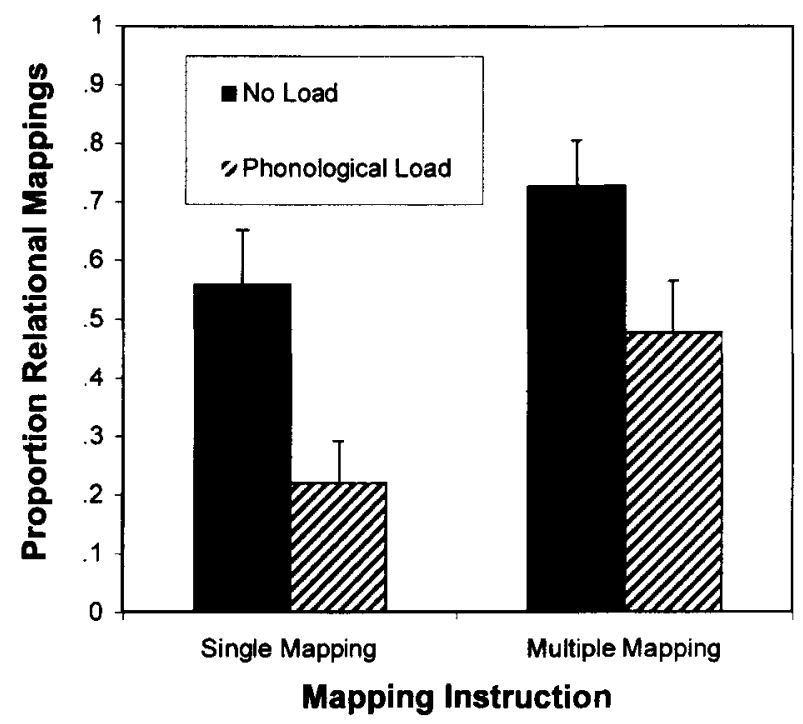

Figure 2. Proportion of relational mappings made by the subjects in Experiment 1, as a function of working-memory load and mapping instruction. Error bars indicate upper bound of $95 \%$ confidence limit. 
the average proportion of digit strings recalled entirely correctly did not differ significantly from . 50 -the figure traditionally viewed as indicating that phonological shortterm memory capacity has been reached. Success rates on the digit-string recall task did not differ significantly as a function of number of objects mapped $[M=.50$, for the single mapping condition vs. $M=.38$, for the multiple mapping condition; $t(21)=1.11, p>.20]$.

The finding of a main effect of working-memory load on the proportion of relational mappings made supports the hypothesis that the process of structure mapping places greater demands on phonological working memory than does the mapping of objects on the basis of attributes. The observation of a main effect of mapping instruction (one vs. three simultaneous mappings) on the proportion of relational mappings replicates the findings of Markman and Gentner (1993), who also found that the 3-map instruction was more likely than the 1-map instruction to prompt subjects to map on the basis of relations between objects.

The lack of a significant interaction between the two independent variables suggests that a phonological workingmemory load influences the tendency to identify relational correspondences in single- and multiple-mapping conditions more or less equally. This finding might be interpreted as indicating that the beneficial effect of simultaneously considering the mappings for three objects is neither amplified nor reduced under conditions of workingmemory load, perhaps because the impact of load on recognition of possible relational mappings is greater in the 1-map condition, but its impact on the mapping process itself is greater in the 3-map condition. However, the fact that a numerical difference (albeit nonsignificant) was observed in performance on the working-memory task between the single- and multiple-mapping conditions raises the possibility that the subjects might have devoted less attention to the working-memory task in the 3 map condition than in the 1-map condition. Thus the absence of a reliable interaction between mapping condition and working-memory load remains open to alternative interpretations.

\section{EXPERIMENT 2}

The purpose of Experiment 2 was to compare the effects of two secondary tasks that were designed to occupy different working-memory systems- the phonological loop and the central executive-that might each be involved in structure mapping. Several theorists (Halford et al., 1998; Waltz et al., 1999) have proposed that, in addition to placing demands on slave systems of working memory, the on-line integration of relations draws upon the function of executive processes controlled by working memory. On the basis of these formulations, we hypothesized that the disruption of executive function due to the requirements of a secondary task would have a negative impact on the process of structure mapping. Accordingly, we predicted that a concurrent task that requires executive control in addition to the phonological loop (generation of random digits) would impair identification of correspondences based on relational similarity more than would a concurrent task that requires only the phonological loop (e.g., repeating a monosyllabic word). As in Experiment 1, we expected that individuals with disrupted phonological-loop function would be less likely to identify correspondences based on relational similarity than those not required to perform a secondary task. In Experiment 2, as in Experiment 1, we examined whether a working-memory load would have differential effects in the single- and multiple-mapping conditions.

\section{Method}

Subjects. One hundred nine undergraduate students at UCLA took part in the experiment in order to receive course credit.

Materials and Apparatus. Macintosh microcomputers running the SuperLab software package were used to present the stimuli. The stimuli included the eight pairs of pictures used by Markman and Gentner (1993), as well as two new pictures of the same type.

Design and Procedure. A $2 \times 3$ between-subjects design was used, with each subject randomly assigned to one of six conditions. The factors manipulated were the type of working-memory distractor task that the subjects were required to perform concurrently with the mapping task (phonological, random-digit generation, or none) and the number of objects in each picture for which the subjects were prompted to identify correspondences (one or three). The phonological distractor involved the subjects' having to repeat the word the continually while performing the mapping task. The random-digit generation task required the subjects to generate random numbers from 1 to 9 while performing the mapping task. Orthogonally, half of the subjects received the 1-map instruction and half received the 3-map instruction. In addition, the picture pairs were presented in one of two different orders, with half the subjects receiving each order.

Each subject sat at a computer with an experimenter. The experimenter read the instructions aloud to each subject. The instructions explained the procedure and the tasks to be performed. The subjects were instructed to point to any object(s) that matched the labeled object(s) so that the experimenter could record their responses. After the experimenter had answered any questions, the experiment began. Prior to the mapping task, the subjects in the two distraction conditions were shown a white screen with a blue star for $1 \mathrm{sec}$, which signaled the subjects to begin performing their respective distractor tasks. The subjects in the distraction conditions were then shown a blank screen for $3 \mathrm{sec}$, during which time they were to continue performing the secondary tasks, followed by the mapping task that was to be performed concurrently with the secondary tasks. In each of the secondary task conditions, the subjects were instructed to maintain a constant rate of vocalization. All the subjects viewed each of the 10 picture pairs once, for as long as they wished. The experimenter recorded the subjects' responses to the mapping questions.

\section{Results and Discussion}

The proportion of relational responses in each distractor condition for both the 1-map and the 3-map conditions is presented in Figure 3. The effects of the type of distractor and type of mapping question on the percentage of relational mappings were analyzed using a $2 \times 3$ between-subjects ANOVA. The ANOVA revealed that the mean proportion of relational responses was significantly higher in the 3-map condition than in the 1map condition $[M=.37$ vs. $M=.25 ; F(1,103)=9.61$, $\left.M S_{\mathrm{e}}=.045, p<.01\right]$. A main effect of distractor task 


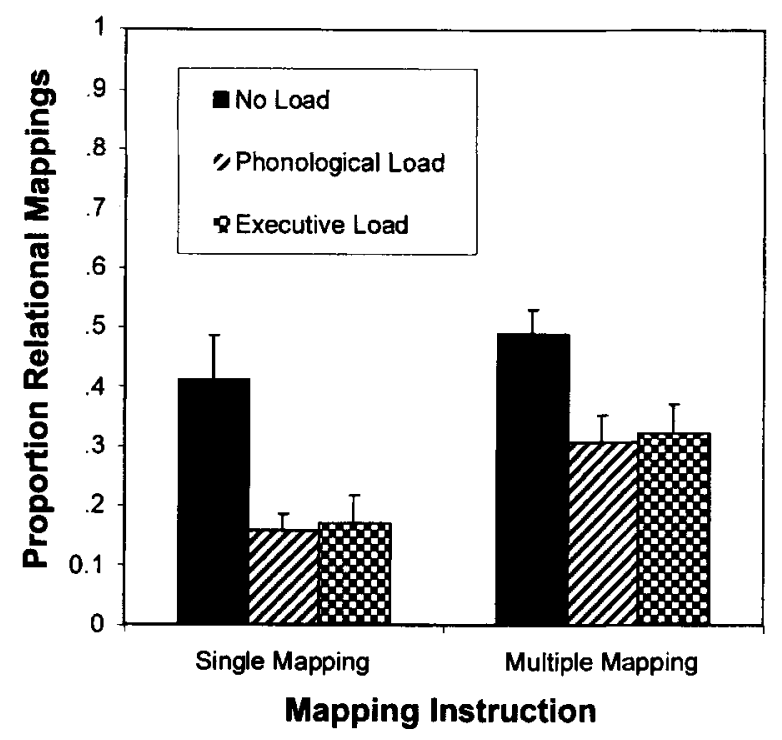

Figure 3. Proportion of relational mappings made by the subjects in Experiment 2, as a function of working-memory load and mapping instruction. Error bars indicate upper bound of $95 \%$ confidence limit.

condition was also obtained $\left[F(2,103)=11.87, M S_{\mathrm{e}}=\right.$ $.045, p<.01]$. A planned comparison revealed that the proportion of relational responses was significantly higher in the no-distraction condition than in the two conditions that received a distractor task $[M=.45$ vs. $M=.24$; $\left.F(1,103)=23.70, M S_{\mathrm{e}}=.045, p<.01\right]$. A further planned comparison revealed that the proportion of relational responses did not differ significantly between the phonological distractor task $(M=.23)$ and random-digit generation $[M=.25 ; F(1,103)<1]$. There was no significant interaction between mapping instruction and distraction condition $[F(2,103)<1]$. As in Experiment 1, only a small percentage $(5 \%)$ of mappings made by the subjects could not be interpreted as either relational or attributional.

The detrimental impact of working-memory load on the proportion of relational mappings made by the subjects supports the hypothesis that the process of structure mapping places greater demands on working memory than does the mapping of objects on the basis of attributes. The observation of a main effect of mapping instruction (one vs. three simultaneous mappings) on the proportion of relational mappings made by the subjects again replicates the findings of Markman and Gentner (1993), who found that the 3-map instructions was much more likely than the 1-map instruction to prompt the subjects to identify relations between objects. In Experiment 2, as in Experiment 1 , no significant interaction was observed between the two independent variables, suggesting that both phonological and executive processes in working memory influence the tendency to identify relational correspondences in single- and multiple-mapping conditions more or less equally.
We obtained no evidence for a differential effect of phonological versus executive working-memory loads; both types of secondary tasks decreased the proportion of relational mapping about equally. We had hypothesized that the executive task would reduce relational responding more than the phonological task would. Although no difference between the two conditions was observed, it is possible that some differential impact occurred in the performance of the secondary task, which was not recorded. In any case, the present results establish that both types of distractor tasks reduce relational responding. The detrimental impact of the phonological task is consistent with evidence obtained by Dunbar and Sussman (1995), who found that impairment on the Wisconsin Card Sorting Test (a standard neuropsychological sign of frontal dysfunction) could be induced in college students by imposing either a phonological workingmemory load or disrupting executive control.

On the other hand, the present results for the phonological task might appear to contradict the results of some other previous studies that used the dual-task paradigm, which suggests that performance on tasks of propositional reasoning is only affected by disruption of the central executive component of working memory (Gilhooly et al., 1993; Toms et al., 1993). The latter studies, however, typically involved explicit visual presentation of sentences to be integrated, which thereby reduced the demands on phonological working memory. By contrast, the mapping task performed by the subjects in our experiment resembles the Wisconsin Card Sort Task in that the mapping task required reasoners to induce the critical semantic relationships from pictures and to hold the relational premises to be integrated in working memory. Accordingly, the present task may be more dependent on the phonological buffer.

\section{GENERAL DISCUSSION}

Using a dual-task paradigm, we examined the role of working memory in the process of structural alignment. We found that people who were prompted to determine correspondences between visual scenes while simultaneously holding information in phonological working memory or performing a task designed to occupy the central executive of working memory identified fewer correspondences on the basis of relational similarity than did those who were not hindered by working-memory loads. Importantly, people who mapped under workingmemory loads did not simply make unsystematic errors; rather, the basis for their mapping responses shifted from relations to attributes.

Our results indicate that the process of structural alignment places greater demands on working memory than does the process of matching attributes. Given that distractor tasks that primarily involve the phonological loop (digit memory in Experiment 1 and repeating the in Experiment 2) decreased relational responding, the firmest 
conclusion is that the phonological buffer is involved in relational processing (encoding and/or mapping). An executive task (the random-digit generation in Experiment 2) also impaired relational responding, but not more than did the phonological task. Further research will be required in order to determine the role of the executive component of working memory and also to investigate the possible additional role of the visuospatial buffer.

Our results do not clearly distinguish whether the negative impact of a memory load is due to its effect on encoding (reduced probability of noticing and representing relations), its effect on the mapping process itself, or both. The present results also do not address the issue of whether the working-memory requirements of on-line relational integration stem specifically from the need to dynamically maintain multiple bindings between objects and conceptual roles, or from the more general need to dynamically maintain a large number of variable bindings. Further research is required in which the nature and number of actively maintained bindings are manipulated independently.

The finding that the process of structural alignment involves increased working-memory demands is consistent with evidence from research in cognitive development that the capacity to represent a system of relations emerges as working-memory capacity increases with age (Halford \& Wilson, 1980). The apparent importance of verbal encoding, even (and perhaps especially) when the stimuli are pictures, is consistent with developmental evidence that learning labels for relations facilitates relational mapping with visual stimuli (Rattermann \& Gentner, 1998). In addition, the present results are consistent with neuropsychological evidence that the capacity to integrate relations is lost as a consequence of damage to prefrontal cortex, which likely reduces working-memory capacity (Waltz et al., 1999). It appears that the representation of a system of relations places demands on working memory because it requires the explicit representation and binding of multiple relations.

The present findings offer support for formulations of cognitive complexity that are specified in terms of relations and suggest that one of the primary effects of factors that reduce available working-memory resources in learning environments is an impairment in the identification and manipulation of relations. Our results have important potential implications for the modeling of reasoning processes, in that they point to working-memory requirements for complex forms of reasoning. Specifically, our findings suggest that models of structure mapping should specify limits on the number and type of role-filler bindings that can simultaneously be represented dynamically. These constraints serve as fundamental assumptions of the LISA model of Hummel and Holyoak (1997), which has been used to simulate a variety of phenomena in analogical mapping and inference (also see Halford et al., 1994). The connection between working memory and the ability to deal with complex cognitive structures may also help us to understand cognitive deficits observed in indi- viduals with focal brain damage or degenerative brain disorders (Waltz et al., 1999) or in normal individuals placed in a state of high anxiety (Tohill \& Holyoak, 2000). A taxonomy of relational complexity may enable the development of testing materials that can be used specifically to assess relational processing capacity in educational as well as neuropsychological settings.

\section{REFERENCES}

Baddeley, A. D. (1986). Working memory. Oxford University Press, Clarendon Press.

Badoeley, A. D. (1992). Working memory. Science, 255, 556-559.

Dunbar, K., \& SusSman, D. (1995). Toward a cognitive account of frontal lobe function: Simulating frontal lobe deficits in normal subjects. In J. Grafman, K. J. Holyoak, \& F. Boller (Eds.), Structure and functions of the human prefrontal cortex (Annals of the New York Academy of Sciences, Vol. 769, pp. 289-304). New York: New York Academy of Sciences.

GENTNER, D. (1983). Structure-mapping: A theoretical framework for analogy. Cognitive Science, 7, 155-170.

Gentner, D., Rattermann, M. J., Markman, A., \& Kotovsky, L. (1995). Two forces in the development of relational similarity. In T. J. Simon \& G. S. Halford (Eds.), Developing cognitive competence: New approaches to process modeling (pp. 263-313). Hillsdale, NJ: Erlbaum.

GENTNER, D., \& TouPIN, C. (1986). Systematicity and surface similarity in the development of analogy. Cognitive Science, 10, 277-300.

Gilhooly, K. J., Logie, R. H., WeTherick, N. E., \& WyNn, V. (1993). Working memory and strategies in syllogistic-reasoning tasks. Memory \& Cognition, 21, 115-124.

Goldstone, R. L., MEdin, D. L., \& Gentner, D. (1991). Relational similarity and the nonindependence of features in similarity judgments. Cognitive Psychology, 23, 222-262.

HALFORD, G. S. (1993). Children's understanding: The development of mental models. Hillsdale, $\mathrm{NJ}$ : Erlbaum.

Halford, G. S., Bain, J. D., \& MaYbery, M. T. (1984). Does a concurrent memory load interfere with reasoning? Current Psychological Research \& Reviews, 3, 14-23.

HALFORD, G. S., \& WILSON, W. H. (1980). A category theory approach to cognitive development. Cognitive Psychology, 12, 356-411.

Halford, G. S., Wilson, W. H., Guo, J., Gayler, R. W., Wiles, J., \& STEWART, J. E. M. (1994). Connectionist implications for processing capacity limitations in analogies. In K. J. Holyoak \& J. A. Barnden (Eds.), Advances in connectionist and neural computation theory: Vol. 2. Analogical connections (pp. 363-415). Norwood, NJ: Ablex.

Halford, G. S., Wilson, W. H., \& Phillips, S. (1998). Processing capacity defined by relational complexity: Implications for comparative, developmental, and cognitive psychology. Behavioral \& Brain Sciences, 21, 803-864.

Hummel, J. E., \& Holyoak, K. J. (1997) Distributed representations of structure: A theory of analogical access and mapping. Psychological Review, 104, 427-466

Klauer, K. C., Stegmaier, R., \& Meiser, T. (1997). Working memory involvement in propositional and spatial reasoning. Thinking \& Reasoning, 3, 9-48.

Markman, A. B., \& Geniner, D. (1993). Structural alignment during similarity comparisons. Cognitive Psychology, 23, 431-467.

Maybery, M. T., Bain, J. D., \& Halford, G. S. (1986). Informationprocessing demands of transitive inference. Journal of Experimental Psychology: Learning, Memory, \& Cognition, 12, 600-613.

Medin, D., Goldstone, R., \& Gentner, D. (1993). Respects for similarity. Psychological Review, 100, 254-278.

PremaCk, D. (1983). The codes of man and beasts. Behavioral \& Brain Sciences, 6, 125-167.

RattermanN, M. J., \& Gentner, D. (1998). The effects of language on similarity: The use of relational labels improves young children's performance in a mapping task. In K. J. Holyoak, D. Gentner, \& B. N. Kokinov (Eds.), Advances in analogy research: Integration of theory 
and data from the cognitive, computational, and neural sciences (pp. 274-282). Sofia, Bulgaria: New Bulgarian University.

Ross, B. H. (1987). This is like that: The use of earlier problems and the separation of similarity effects. Journal of Experimental Psychology: Learning, Memory, \& Cognition, 13, 629-639.

Ross, B. H. (1989). Distinguishing types of superficial similarities: Different effects on the access and use of earlier problems. Journal of Experimental Psychology: Learning, Memory, \& Cognition, 15, 456-468. SMITH, L. B. (1989). From global similarities to kinds of similarities The construction of dimensions in development. In S. Vosniadou \& A. Ortony (Eds.), Similarity and analogical reasoning (pp. 146-178). New York: Cambridge University Press.

Thompson, R. K. R., OdEN, D. L., \& Boysen, S. T. (1997). Languagenaive chimpanzees (Pan troglodytes) judge relations between relations in a conceptual matching-to-sample task. Journal of Experimental Psychology: Animal Behavior Processes, 23, 31-43.
TOHILl, J. M., \& HoLYoAK, K. J. (2000). The impact of anxiety on analogical reasoning. Thinking \& Reasoning, 6, 27-40.

TOMS, M., MorRIS, N., \& WARD, D. (1993). Working memory and conditional reasoning. Quarterly Journal of Experimental Psychology, 46A, 469-699.

Tversky, A. (1977). Features of similarity. Psychological Review, 84, 327-352.

Waltz, J. A., Knowlton, B. J., Holyoak, K. J., Boone, K. B., Mishkin, F. S., de Menezes Santos, M., Thomas, C. R., \& Miller, B. L. (1999). A system for relational reasoning in human prefrontal cortex. Psychological Science, 10, 119-125.

(Manuscript received March 3, 1999; revision accepted for publication November 9,1999 .) 